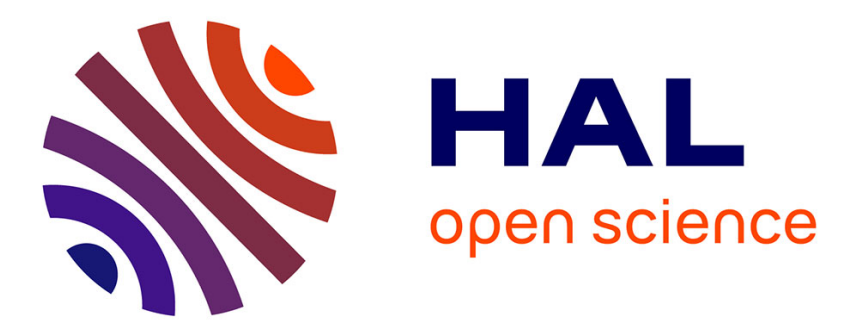

\title{
New Low-Rank Filters for MIMO-STAP based on an Orthogonal Tensorial Decomposition
}

\author{
Frédéric Brigui, Maxime Boizard, Guillaume Ginolhac, Fréderic Pascal
}

\section{To cite this version:}

Frédéric Brigui, Maxime Boizard, Guillaume Ginolhac, Fréderic Pascal. New Low-Rank Filters for MIMO-STAP based on an Orthogonal Tensorial Decomposition. IEEE Transactions on Aerospace and Electronic Systems, 2018, 54 (3), pp.1208-1220. 10.1109/TAES.2017.2776679 . hal-01789076

\section{HAL Id: hal-01789076 \\ https://hal.science/hal-01789076}

Submitted on 26 Feb 2020

HAL is a multi-disciplinary open access archive for the deposit and dissemination of scientific research documents, whether they are published or not. The documents may come from teaching and research institutions in France or abroad, or from public or private research centers.
L'archive ouverte pluridisciplinaire HAL, est destinée au dépôt et à la diffusion de documents scientifiques de niveau recherche, publiés ou non, émanant des établissements d'enseignement et de recherche français ou étrangers, des laboratoires publics ou privés. 


\title{
New Low-Rank Filters for MIMO-STAP based on an Orthogonal Tensorial Decomposition
}

Frédéric Brigui, Maxime Boizard, Guillaume Ginolhac Member, IEEE, Frédéric Pascal Senior Member, IEEE

\begin{abstract}
We develop in this paper a new adaptive LR filter for MIMO-STAP application based on a tensorial modelling of the data. This filter is based on an extension of the HOSVD (which is also one possible extension of SVD to the tensor case), called AU-HOSVD, which allows to consider the combinations of dimensions. This property is necessary to keep the advantages of the STAP and the MIMO characteristics of the data. We show that the choice of a good partition (as well as the tensorial modelling) is not heuristic but have to follow several features. Thanks to the derivation of the theoretical formulation of multimode ranks for all partitions, the tensorial LR filters are easy to compute. Results on simulated data show the good performance of the AU-HOSVD LR filters in terms of secondary data and clutter notch.
\end{abstract}

\section{Index Terms}

Orthogonal Tensor Decomposition, MIMO, STAP, Low Rank Clutter, Radar

\section{INTRODUCTION}

Space Time Adaptive Processing (STAP) is a technique used in airborne radar to detect moving target embedded in an interference background such as jamming or strong clutter [1], [2]. While conventional radars are capable of detecting targets both in the fast-time domain related to target range and in the slow-time domain related to target velocity, STAP uses an additional domain (space)

Frédéric Brigui is with NTU-Temasek Lab., email: frederic.brigui@gmail.com.

Maxime Boizard is with SATIE-ENS Cachan, email: maxime.boizard@satie.ens-cachan.fr.

Guillaume Ginolhac is with LISTIC - Université Savoie Mont-Blanc, email: guillaume.ginolhac@univ-smb.fr.

Frederic Pascal is with LSS - CentraleSupelec, email:frederic.pascal@ supelec.fr.

The work of F. Pascal has been partially supported by the DGA grant Nř 2013.60.0011.00.470.75.01. 
related to the target angular localization. The consequence is a two-dimensional adaptive filtering technique which uses jointly temporal and spatial dimensions to cancel interference and to improve target detection. STAP has been firstly developed for conventional phased array (or SIMO for SingleInput Multiple-Output) with spatial dimension corresponding to receive array and temporal dimension corresponding to pulses. Recently, more complex radar systems have emerged with MIMO radars [3], [4]. Compared to conventional phased array, MIMO radar offers additional degrees of freedom by considering multiple transmitters and multiple receivers. Several MIMO configurations have been proposed in the literature but we focus in this study on the coherent and colocated MIMO [5]. In this configuration, transmit antennas emit orthogonal waveforms. Moreover the transmit and the receive arrays are closely located to ensure that the received signal from any scatterer is coherent between the pairs of transmit and receive elements. Thereby, higher spatial resolution can be achieved. STAP has been naturally extended to coherent MIMO radar [6], [7]. Compared to SIMO STAP, the increased angular resolution allows narrower clutter suppression for MIMO radar.

In side-looking STAP (as considered in this paper), the ground clutter is shown to have a Low Rank (LR) structure for both SIMO and MIMO radars. The LR structure of the clutter can be exploited to derive STAP methods to effectively suppress clutter [8], [9]. For fully adaptive STAP, the filter is derived by inverting the full-dimension clutter covariance matrix (CCM). To achieve good performances, the estimation of the CCM requires a number of independent and identically distributed (i.i.d) secondary data equal to two times of the dimension of the data, which is practically not possible. Instead of inverting the CCM, LR STAP methods are based on the use of the projector on the LR clutter subspace to remove the clutter contribution. LR STAP methods allow then to reduce the number of secondary data to achieve the same performance as the fully adaptive STAP [8], [9], [10], [11] (i.e. $2 r$ where $r$ is the clutter rank which is usually greatly lower than the data dimension). The estimation of the clutter subspace projector is done by using the SVD (Singular Value Decomposition) of the Sample Covariance Matrix (SCM) which is built from secondary data.

The reduction of the secondary support for LR STAP filter clearly depends on the rank of the CCM. In turn, the CCM rank depends on the studied physical phenomenon, on the radar configuration and on the way the data are arranged. Since the physical phenomenon and the radar configuration are fixed, we propose in this study to focus on the data arrangement to further exploit the LR property of the clutter. As for most STAP methods, LR filters are vectorial techniques. The specific multidimensional features of MIMO data are then not fully exploited. MIMO data vectorial representation can be seen as a unique and limited way to structure data. Another way to arrange multidimensional data is to order them by using tensor which are multidimensional arrays. Tensors preserve data structure in the sense that the ordering is not fixed. Multilinear algebra [12], [13] provides a good framework to exploit multidimensional data represented by tensors. However, generalizing matrix-based algorithms 
to the multilinear algebra framework is not a trivial task. Specifically LR filters are based on the SVD in linear algebra. But there is no single multilinear extension of the SVD, with exactly the same properties as the SVD. Depending on which properties are preserved, several extensions of the SVD have been introduced. However, two main decompositions exist: CANDECOMP/PARAFAC (CP) [14], which conserves the rank properties of SVD and the identifiability properties, and the Higher Order Singular Value Decomposition (HOSVD) [13] derived from the Tucker decomposition [15], which keeps the orthogonality properties. To derive LR filters, orthogonal properties are needed and fir that, the family of HOSVD decompositions are used in this paper. The basic HOSVD is based on the classic tensor unfolding. This unfolding transforms a tensor into a matrix in order to highlight each dimension separately. HOSVD is thus shown to be inefficient to design LR-filters for multidimensional STAP systems because the LR structure is contained into the combination of time and space dimension. In order to exploit the structure of multidimensional STAP data, [16], [17] proposed a new set of orthogonal decompositions, the Alternative Unfolding HOSVD (AU-HOSVD). A tensor can be represented by different unfoldings according to the considered partitioning and each unfolding is a set of partitions. The AU-HOSVD can be seen as a generalization of the HOSVD for any unfolding of a tensor. In this way, the classic tensor unfolding associated to HOSVD is seen as a particular multidimensional representation in which each partition is formed by one of the dimensions of the tensor. In the same way, the vectorial representation associated to the SVD is a particular unfolding for which only one single partition is considered. These two data configurations are extreme cases in which the elements are either combined over all dimensions or separated for each dimension. However many sets of partitions lie in between these two extremes.

Based on the AU-HOSVD framework, we propose to develop tensorial LR STAP filters for MIMOSTAP radars. LR STAP filters based on AU-HOSVD have already been considered for polarimetric STAP in [16], [17] and showed good results in terms of reduction of the number of secondary data. Nevertheless, we showed in these previous papers that AU-HOSVD leads to several tensorial LR filters which are not all interesting for the considered application. The choice of the correct LR filters is a difficult task. In this paper, we propose to derive tensorial LR STAP filters for MIMO-STAP by giving a solid framework to select the correct tensorial LR filters. This framework is based on the clutter characteristics described by the clutter covariance tensor (CCT) [18]. We rely on 3 features of the CCT to build our selection framework:

- $F_{1}$ : Space-Time (ST) combination because STAP processing is based on the combination of space and time dimensions.

- $F_{2}$ : Virtual Antenna (VA) because in coherent MIMO, resolution is improved through the combination of the transmit and receive arrays that creates a virtual antenna. 
- $F_{3}$ : LR property of the clutter covariance tensor (CCT) since it is essential to derive efficient LR filters. It is important to have the smallest multimode ranks (ranks of all possible unfoldings of the CCT for a corresponding partition) in particular with respect of the total size of the data. The two first features have been used to justify the AU-HOSVD using instead of the HOSVD. Moreover, they will be useful to select the correct combination of dimensions. The third one will be used to propose a 4-th order tensor to model the MIMO-STAP data. By nature MIMO-STAP data are multidimensional, with at least 3 dimensions, spatial transmit and receive and Doppler. But, since the number of pulses is often large which leads to high CCT multimode ranks, arranging the data in sets of sub-pulses will allow to fulfil feature $F_{3}$. We will notice that this derivation is inspired by the pre-Doppler approaches developed for STAP [19], [20]. Finally, we give the theoretical formulation of the CCT ranks for the different possible unfoldings of the tensor model. Indeed, CCT multimode ranks which are composed of the ranks of each partition for a given unfolding, are required to derived tensorial LR STAP filters. In vectorial case the rank of the CCM is given by Brennan's rule [21] for SIMO and Generalized Brennan's rule [6] for MIMO but these rules do not apply to others data configurations. Based on the following studies of rank derivation [22][23], we derive rank formula for all the possible data configuration associated to the multiple LR filters based on the AU-HOSVD. The performances of the LR filter for the MIMO-STAP data are first evaluated on simulated data with orthogonal waveforms. Finally a simulation with realistic waveforms will be tested and shows the robustness of the proposed approach.

The paper is organized as follows. Section II gives the mathematical background of the tensor decompositions, especially of the AU-HOSVD. We present in Section III the tensorial LR STAP filters for MIMO-STAP based the AU-HOSVD with the tensor model and the corresponding partitions. Finally in Section IV we evaluate the performances of the proposed tensorial LR STAP filters for MIMO-STAP. Section V is dedicated to simulation with real MIMO waveforms.

The following convention is adopted: a denotes a vector, $\mathbf{A}$ denotes a matrix and $\mathcal{A}$ denotes a tensor. $\mathbf{A}^{T}$ denotes the transpose operator, $\mathbf{A}^{*}$ the conjugate and $\mathbf{A}^{H}$ the transpose conjugate. $\mathbf{I}_{N}$ is the identity matrix of size $N \times N \cdot \mathcal{C N}(\mathbf{a}, \mathbf{R})$ is a complex Gaussian vector of mean a and of covariance matrix $\mathbf{R}$. The expectation is denoted by $E[$.] and the Frobenius norm by $\|$.$\| .$

\section{Alternative Unfolding Higher Order Singular Decomposition (AU-HOSVD)}

In this section, we present a brief overview of the HOSVD and the new decomposition, the AUHOSVD. More details on the AU-HOSVD and its application to polarimetric STAP can be found in [16], [17].

Let $\mathcal{H}, \mathcal{B} \in \mathbb{C}^{I_{1} \times \ldots \times I_{P}}$, be two $P$ th order tensors and $h_{i_{1}, \ldots, i_{p}}, b_{i_{1}, \ldots, i_{p}}$ their elements. 


\section{A. Classical Tools of Multilinear Algebra}

Unfoldings: In this paper, three existing unfoldings are used; for a general definition of tensor unfolding, we refer the reader to [12].

- vector: vec transforms a tensor $\mathcal{H}$ into a vector, $\operatorname{vec}(\mathcal{H}) \in \mathbb{C}^{I_{1} I_{2} \ldots I_{P}}$. We denote $v e c^{-1}$, the inverse operator.

- matrix: this operator transforms the tensor $\mathcal{H}$ into a matrix $[\mathcal{H}]_{p} \in \mathbb{C}^{I_{p} \times I_{1} \ldots I_{p-1} I_{p+1} \ldots I_{P}}, p=$ $1 \ldots P$. For example, $[\mathcal{H}]_{1} \in \mathbb{C}^{I_{1} \times I_{2} \ldots I_{P}}$. This transformation allows to enhance simple information (i.e. information contained in one dimension of the tensor).

- square matrix: this operator transforms the square tensor $\mathcal{R} \in \mathbb{C}^{I_{1} \times I_{2} \ldots \times I_{P} \times I_{1} \times I_{2} \ldots \times I_{P}}$ into a square matrix, $\operatorname{SqMat}(\mathcal{R}) \in \mathbb{C}^{I_{1} \ldots I_{P} \times I_{1} \ldots I_{P}} . S q M a t^{-1}$ is the inverse operator.

The inverse operators always exists. However, the way the tensor is unfolded must be known.

\section{Products:}

- The scalar product $<\mathcal{H}, \mathcal{B}>$ of two tensors is defined as:

$$
\begin{aligned}
<\mathcal{H}, \mathcal{B}> & =\sum_{i_{1}} \sum_{i_{2}} \ldots \sum_{i_{P}} b_{i_{1} i_{2} \ldots i_{P}}^{*} h_{i_{1} i_{2} \ldots i_{P}} \\
& =\operatorname{vec}(\mathcal{B})^{H} \operatorname{vec}(\mathcal{H}) .
\end{aligned}
$$

It is the natural extension of the classical scalar product.

- Let $\mathbf{E} \in \mathbb{C}^{J_{n} \times I_{n}}$ be a matrix, the $n$-mode product between $\mathbf{E}$ and a tensor $\mathcal{H}$ is defined as:

$$
\begin{array}{rrr} 
& \mathcal{G}=\mathcal{H} \times{ }_{n} \mathbf{E} \in \mathbb{C}^{I_{1} \times \ldots \times J_{n} \times \ldots \times I_{P}} \\
\Longleftrightarrow & (\mathcal{G})_{i_{1} \ldots j_{n} \ldots i_{P}}=\sum_{i_{n}} h_{i_{1} \ldots i_{n} \ldots i_{P}} e_{j_{n} i_{n}} \\
& \Longleftrightarrow & {[\mathcal{G}]_{n}=\mathbf{E}[\mathcal{H}]_{n}}
\end{array}
$$

- The outer product between $\mathcal{H}$ and $\mathcal{B}, \mathcal{E}=\mathcal{H} \circ \mathcal{B} \in \mathbb{C}^{I_{1} \times \ldots \times I_{P} \times I_{1} \times \ldots \times I_{P}}$ is defined as:

$$
e_{i_{1} \ldots i_{P} i_{1} \ldots i_{P}}=h_{i_{1} \ldots i_{P}} \cdot b_{i_{1} \ldots i_{P}}
$$

Rank definition: There are two concepts to define the rank of a tensor:

- the tensor rank, which is defined as the minimum number of rank-1 tensors necessary to obtain the considered tensor. This rank is impossible to compute in general cases and it will not be used in the sequel.

- the $p$-ranks which are defined as the ranks of the unfolding of the tensor, $r_{p}=\operatorname{rank}\left([\mathcal{H}]_{p}\right)$. 


\section{B. Higher Order Singular Value Decomposition}

The Higher Order Singular Value Decomposition (HOSVD) is particular case of Tucker decomposition [15] with orthogonality properties. HOSVD decomposes a tensor $\mathcal{H} \in \mathbb{C}^{I_{1} \times \ldots \times I_{P}}$ as follows [13]:

$$
\mathcal{H}=\mathcal{K} \times{ }_{1} \mathbf{U}^{(1)} \ldots \times_{P} \mathbf{U}^{(P)},
$$

where $\forall n, \mathbf{U}^{(n)} \in \mathbb{C}^{I_{n} \times I_{n}}$ is an orthonormal matrix and $\mathcal{K} \in \mathbb{C}^{I_{1} \times \ldots \times I_{P}}$ is the core tensor, which satisfies the all-orthogonality conditions [13]. The matrix $\mathbf{U}^{(n)}$ is given by the Singular Value Decomposition of the $n$-dimension unfolding tensor, $[\mathcal{H}]_{n}=\mathbf{U}^{(n)} \boldsymbol{\Sigma}^{(n)} \mathbf{V}^{(n) H}$.

Remark: Let $\mathcal{H} \in \mathbb{C}^{I_{1} \times I_{2} \ldots \times I_{P} \times I_{1} \times I_{2} \ldots \times I_{P}}$ be a $2 P$ th order Hermitian tensor, i.e $h_{i_{1}, \ldots, i_{p}, j_{1}, \ldots, j_{p}}=$ $h_{j_{1}, \ldots, j_{p}, i_{1}, \ldots, i_{p}}^{*}$. The HOSVD of $\mathcal{H}$ is written as [18]:

$$
\mathcal{H}=\mathcal{K} \times{ }_{1} \mathbf{U}^{(1)} \ldots \times_{P} \mathbf{U}^{(P)} \times_{P+1} \mathbf{U}^{(1) *} \ldots \times_{2 P} \mathbf{U}^{(P) *}
$$

The first two conditions $F_{1}$ and $F_{2}$ require to build algorithms with combined dimensions. By using classical unfolding, one can notice that the HOSVD only considers the simple information. However, in STAP and in order to remove properly the clutter and to detect slow-moving targets, the combination of the spatial and temporal dimensions is needed even in a tensorial approach [16], [17]. Therefore, the HOSVD is useless for STAP or MIMO-STAP applications to derive LR filters. In order to use some combinations of dimensions and to respect the features $F_{1}$ and $F_{2}$, it is appropriate to use another orthogonal decomposition called the AU-HOSVD [16], [17], that generalizes the HOSVD. First, let us define new operators involved in the AU-HOSVD definition.

\section{Extension of multilinear algebra tools}

Notation of indices: In order to consider correlated information, we introduce a new notation for the indices of a tensor. We consider $\mathcal{H} \in \mathbb{C}^{I_{1} \times \ldots \times I_{P}}$, a $P$ th order tensor. We denote $\mathbb{A}=\{1, \ldots, P\}$ the set of the dimensions and $\mathbb{A}_{1}, \ldots, \mathbb{A}_{Q}, Q$ subsets of $\mathbb{A}$ which define a partition of $\mathbb{A}$. In other words, $\mathbb{A}_{1}, \ldots, \mathbb{A}_{Q}$ satisfy the following conditions:

- $\mathbb{A}_{1} \cup \ldots \cup \mathbb{A}_{Q}=\mathbb{A}$

- They are pairwise disjoint, i.e. $\forall i \neq j, \mathbb{A}_{i} \cap \mathbb{A}_{j}=\emptyset$.

Moreover $\mathbb{C}^{I_{1} \ldots I_{P}}$ is denoted $\mathbb{C}^{I_{\AA}}$. For example, when $\mathbb{A}_{1}=\{1,2\}$ and $\mathbb{A}_{2}=\{3,4\}, \mathbb{C}^{I_{\mathbb{A}_{1}} \times I_{\mathbb{A}_{2}}}$ means $\mathbb{C}^{I_{1} I_{2} \times I_{3} I_{4}}$.

A generalization of unfolding in matrices: In order to build our new decomposition, we need a generalized unfolding, adapted from [12]. This operator allows to unfold a tensor into a matrix whose dimensions could be any combination $\mathbb{A}_{q}$ of the tensor dimensions. It is denoted as $[.]_{\mathbb{A}_{q}}$ and it transforms $\mathcal{H}$ into a matrix $[\mathcal{H}]_{\mathbb{A}_{q}} \in \mathbb{C}^{I_{\mathbb{A}_{q}} \times I_{\mathbb{A}_{\backslash} \mathbb{A}_{q}}}$. 
A new unfolding in tensors: We denote as Reshape the operator which transforms a tensor $\mathcal{H}$ into another tensor $\operatorname{Reshape}\left(\mathcal{H}, \mathbb{A}_{1}, \ldots, \mathbb{A}_{Q}\right) \in \mathbb{C}^{I_{\mathbb{A}_{1}} \times \ldots \times I_{\mathbb{A}_{Q}}}$ and Reshape ${ }^{-1}$ the inverse operator.

A new tensor product: The $n$-mode product allows to multiply a tensor with a matrix along 1 dimension. We propose to extend the $n$-mode product to multiply a tensor with a matrix along several dimensions, combined in $\mathbb{A}_{q}$. Let $\mathbf{D} \in \mathbb{C}^{I_{\mathbb{A}_{q}} \times I_{\mathbb{A}_{q}}}$ be a square matrix. This new product, called multimode product is defined as:

$$
\mathcal{B}=\mathcal{H} \times_{\mathbb{A}_{q}} \mathbf{D} \Longleftrightarrow[\mathcal{B}]_{\mathbb{A}_{q}}=\mathbf{D}[\mathcal{H}]_{\mathbb{A}_{q}}
$$

Multimode ranks: For a given partition $\mathbb{A}_{1}, \ldots, \mathbb{A}_{Q}$, the multimode ranks $r_{\mathbb{A}_{1}}, \ldots, r_{\mathbb{A}_{Q}}$ are defined as the ranks of the unfoldings of the tensor, $r_{\mathbb{A}_{q}}=\operatorname{rank}\left([\mathcal{H}]_{\mathbb{A}_{q}}\right)$.

\section{Alternative Unfolding HOSVD}

Definition: Let $\mathcal{H} \in \mathbb{C}^{I_{1} \times \ldots \times I_{P}}$ and $\mathbb{A}_{1} \ldots \mathbb{A}_{Q}$ a partition of $\mathbb{A}$. Then $\mathcal{H}$ may be decomposed as follows:

$$
\mathcal{H}=\mathcal{K}_{\mathbb{A}_{1} / \ldots / \mathbb{A}_{Q}} \times_{\mathbb{A}_{1}} \mathbf{U}^{\left(\mathbb{A}_{1}\right)} \ldots \times_{\mathbb{A}_{Q}} \mathbf{U}^{\left(\mathbb{A}_{Q}\right)},
$$

where:

- $\forall q \in[1, Q], \mathbf{U}^{\left(\mathbb{A}_{q}\right)} \in \mathbb{C}^{\mathbb{A}_{q} \times \mathbb{A}_{q}}$ is unitary and is computed from the SVD of the $\mathbb{A}_{q}$-dimension unfolding $[\mathcal{H}]_{\mathbb{A}_{q}}$.

- $\mathcal{K}_{\mathbb{A}_{1} / \ldots / \mathbb{A}_{Q}} \in \mathbb{C}^{I_{1} \times \ldots \times I_{P}}$ is the core tensor. It has the same properties as the HOSVD core tensor. Notice that there are several ways to decompose a tensor with the AU-HOSVD. Each choice of the $\mathbb{A}_{1}, \ldots, \mathbb{A}_{Q}$ gives a different decomposition. For a $P$ th order tensor the number of different $\mathrm{AU}-\mathrm{HOSVD}$ is given by the Bell number, $B_{P}$ :

$$
\begin{aligned}
B_{1} & =1 \\
B_{P+1} & =\sum_{k=1}^{P}\left(\begin{array}{l}
P \\
k
\end{array}\right) B_{k}
\end{aligned}
$$

Remark: Let $\mathcal{H} \in \mathbb{C}^{I_{1} \times I_{2} \ldots \times I_{P} \times I_{1} \times I_{2} \ldots \times I_{P}}$ be a $2 P$ th order Hermitian tensor. We consider $2 Q$ subsets of $\left\{I_{1}, \ldots, I_{P}, I_{1}, \ldots, I_{P}\right\}$ such as:

- $\mathbb{A}_{1}, \ldots, \mathbb{A}_{Q}$ and $\mathbb{A}_{Q+1}, \ldots, \mathbb{A}_{2 Q}$ are two partitions of $\left\{I_{1}, \ldots, I_{P}\right\}$

- $\forall q \in[1, Q], \mathbb{A}_{q}=\mathbb{A}_{q+Q}$

Under these conditions, the AU-HOSVD of $\mathcal{H}$ is written:

$$
\mathcal{H}=\mathcal{K}_{\mathbb{A}_{1} / \ldots / \mathbb{A}_{2 Q}} \times_{\mathbb{A}_{1}} \mathbf{U}^{\left(\mathbb{A}_{1}\right)} \ldots \times_{\mathbb{A}_{Q}} \mathbf{U}^{\left(\mathbb{A}_{Q}\right)} \times_{\mathbb{A}_{Q+1}} \mathbf{U}^{\left(\mathbb{A}_{1}\right) *} \ldots \times_{\mathbb{A}_{2 Q}} \mathbf{U}^{\left(\mathbb{A}_{Q}\right) *}
$$


Extension of the orthogonal projector: As discussed in the introduction, the main motivation for introducing the AU-HOSVD is to extract the combined information when processing the low-rank decomposition. Let $\mathcal{H}, \mathcal{H}_{c}, \mathcal{H}_{0}$ be three Pth order tensors such that:

$$
\mathcal{H}=\mathcal{H}_{c}+\mathcal{H}_{0}
$$

where $\mathcal{H}_{c}$ is a $\left(r_{\mathbb{A}_{1}}, \ldots, r_{\mathbb{A}_{Q}}\right)$ low rank tensor ${ }^{1}\left(r_{\mathbb{A}_{q}}=\operatorname{rank}\left(\left[\mathcal{H}_{c}\right]_{\mathbb{A}_{q}}\right)\right)$. Then $\mathcal{H}_{0}$ is approximated by:

$$
\mathcal{H}_{0} \approx \mathcal{H} \times_{\mathbb{A}_{1}} \mathbf{U}_{0}^{\left(\mathbb{A}_{1}\right)} \mathbf{U}_{0}^{\left(\mathbb{A}_{1}\right) H} \ldots \times_{\mathbb{A}_{Q}} \mathbf{U}_{0}^{\left(\mathbb{A}_{Q}\right)} \mathbf{U}_{0}^{\left(\mathbb{A}_{Q}\right) H}
$$

where $\mathbf{U}_{0}^{\left(\mathbb{A}_{1}\right)}, \ldots, \mathbf{U}_{0}^{\left(\mathbb{A}_{Q}\right)}$ minimize the following criterion:

$$
\left(\mathbf{U}_{0}^{\left(\mathbb{A}_{1}\right)}, \ldots, \mathbf{U}_{0}^{\left(\mathbb{A}_{Q}\right)}\right)=\operatorname{argmin}\left\|\mathcal{H}_{0}-\mathcal{H} \times_{\mathbb{A}_{1}} \mathbf{U}_{0}^{\left(\mathbb{A}_{1}\right)} \mathbf{U}_{0}^{\left(\mathbb{A}_{1}\right) H} \ldots \times_{\mathbb{A}_{Q}} \mathbf{U}_{0}^{\left(\mathbb{A}_{Q}\right)} \mathbf{U}_{0}^{\left(\mathbb{A}_{Q}\right) H}\right\|^{2}
$$

In this paper we use a truncation of $\mathbf{U}^{\left(\mathbb{A}_{q}\right)}$ for $\mathbf{U}_{0}^{\left(\mathbb{A}_{q}\right)}$, i.e. $\mathbf{U}_{0}^{\left(\mathbb{A}_{q}\right)}=\left[\mathbf{u}_{r_{\mathbb{A}_{q}}+1}^{\left(\mathbb{A}_{1}\right)} \ldots \mathbf{u}_{\mathbb{A}_{q}}^{\left(\mathbb{A}_{q}\right)}\right]$. This solution is not optimal in the sense of least squares. However like for HOSVD, it is a correct approximation(see [18], [24]) in most cases. Moreover it is easy to implement. That is why iterative algorithms will not be used in this paper ${ }^{2}$.

\section{MIMO-STAP BASED ON THE AU-HOSVD}

\section{A. Tensorial Signal Model}

1) MIMO Radar Configuration: We consider a MIMO radar with $M$ transmit antennas spaced by $d_{e}$ and $N$ receive antennas spaced by $d_{r}$. We assume that the radar system is in the coherent and colocated MIMO configuration [4], [25], [5], i.e. the transmit and the receive arrays are closely located to ensure that the received signal is coherent between the pairs of transmit and receive elements (Fig. 1). We also consider $L$ pulses with a pulse repetition interval $T_{r}$. The wavelength of transmit signals is denoted by $\lambda_{0}$ and the speed of the airborne platform by $v_{a}$. The radar is in a side-looking (SL) configuration. We assume that the $M$ waveforms are ideally orthogonal and can then be perfectly disentangle at each receiver ${ }^{3}$. In addition of the cell under test (CUT), we assume that $K$ observations are available. We assume that the CUT and the $K$ observation cells are independent and identically distributed.

\footnotetext{
${ }^{1}$ This definition of rank is directly extended from the definition of $p$-ranks.

${ }^{2}$ The partition of the tensor does not guarantee that each subset is low rank. In such case, the result of the AU-HOSVD is the same as for vectorial approach using SVD. Nevertheless, most of the possible partitions of the tensor give low-rank subsets.

${ }^{3}$ This assumption is made to develop the different filters. We will evaluate the proposed filters on realistic simulated MIMO data in Section V.
} 
2) Choice of the Tensor Model: Before the choice of the tensor model, we investigate the possible performance reached by the tensorial LR filters. A common way to evaluate the performances of an adaptive filter, is the Signal to Interference plus Noise Ratio (SINR) loss:

$$
\rho=\frac{S I N R_{\text {out }}}{S I N R_{\max }}
$$

where $S I N R_{\text {out }}$ is the SINR at the output of the adaptive STAP filter and $S I N R_{\max }$ the SINR at the output of the optimal filter [1]. One way to study $\rho$ is to theoretically derive its distribution like in [10] for Gaussian noise or in [26] for non Gaussian noise. These derivations are based on a perturbation analysis of the projector onto the clutter subspace. For the vectorial LR STAP filter, the expectation of the theoretical SINR loss is given by [10]:

$$
E[\rho]=1-\frac{r}{K},
$$

where $r$ is the clutter rank. A $3 \mathrm{~dB}$ loss is then reached for $K=2 r$. This allows to conclude that the value of rank $r$ is really important to reach the best SINR loss performance. Even if the theoretical SINR loss is not known for the tensorial LR filters, it seems that it depends on the multimode ranks (defined in the subsection II-C). Therefore, this analysis allows to understand the feature $F_{3}$ which states that the clutter subspace has to be low-rank.

The most general model is to consider a tensor of order $M N L$ for the data (which generates a covariance tensor of order $M N L \times M N L)$. Studying the $M N L$-th order data tensor or the $M N L \times$ $M N L$-th order CCT is thus equivalent. Nevertheless, since $M N L$ could be large, it is interesting to add a degree of liberty in order to respect the third condition of LR structure for the clutter contribution. In MIMO-STAP, the spatial parameters $M$ and $N$ are often small whereas the number of pulses $L$ can be important. Therefore, we propose to segment the pulses dimension into small subsets of size $L_{1}\left(L_{1}<L\right)$ with a number of subsets equal to $L_{2}\left(L_{2}=L / L_{1}\right)$. This operation is possible in MIMO-STAP thanks to an interesting property of the clutter: it is stationary over the overall CPI ${ }^{4}$. Note that this property for a stationary clutter has been used to design pre-Doppler approaches for STAP in [2], [20] in order to reduce the computational complexity and to reduce the number of secondary data. Pre-Doppler adaptive STAP weights are computed for a reduced sub-pulse sets and then filtering is performed coherently using outputs from all the subsets. The size of the sub-pulse set is usually 2 or 3 which allows both STAP processing and clutter stationary property.

To conclude, we propose to model the MIMO-STAP received data as a 4-th order tensor $X \in$ $\mathbb{C}^{N \times M \times L_{1} \times L_{2}}$.

${ }^{4}$ Other clutter properties can be used to derive different models. For example, the clutter in SL configuration is known to be low correlated in angle and Doppler [27], [28]. 
3) Model of the received data: The received signal model of a target of the CUT located at the spatial and Doppler frequencies $\left(f_{s}, f_{d}\right)$, can be then written as follows:

$$
\begin{aligned}
& \boldsymbol{x}=\alpha \boldsymbol{S}\left(f_{s}, f_{d}\right)+\mathcal{C}+\mathcal{N} \\
& \boldsymbol{x}_{k}=\boldsymbol{e}_{k}+\mathcal{N}_{k}
\end{aligned} \quad k \in[1, K],
$$

where $\boldsymbol{X}, \boldsymbol{X}_{k} \in \mathbb{C}^{N \times M \times L_{1} \times L_{2}}$ are the received signal tensors. $\mathcal{S}\left(f_{s}, f_{d}\right) \in \mathbb{C}^{N \times M \times L_{1} \times L_{2}}$ is the steering tensor and is derived as follows:

$$
\boldsymbol{S}\left(f_{s}, f_{d}\right)=\mathbf{a}_{R x}\left(f_{s}\right) \circ \mathbf{a}_{T x}\left(f_{s}\right) \circ \mathbf{a}_{L_{1}}\left(f_{d}\right) \circ \mathbf{a}_{L_{2}}\left(f_{d}\right),
$$

where,

- $\mathbf{a}_{R x}\left(f_{s}\right)=\left[1 \exp \left(j 2 \pi f_{s}\right) \ldots \exp \left(j 2 \pi(N-1) f_{s}\right)\right]^{T}$ and $\mathbf{a}_{T x}\left(f_{s}\right)=\left[1 \exp \left(j 2 \pi \gamma f_{s}\right) \ldots \exp (j 2 \pi \gamma(M-\right.$

1) $\left.\left.f_{s}\right)\right]^{T}$ are respectively the receive and the transmit spatial steering vectors.

- $\mathbf{a}_{L_{1}}\left(f_{d}\right)=\left[1 \exp \left(j 2 \pi f_{d} T_{r}\right) \ldots \exp \left(j 2 \pi\left(L_{1}-1\right) f_{d} T_{r}\right)\right]^{T}$ and $\mathbf{a}_{L_{2}}\left(f_{d}\right)=\left[1 \exp \left(j 2 \pi f_{d} L_{1} T_{r}\right) \ldots \exp \left(j 2 \pi\left(L_{2}-\right.\right.\right.$

1) $\left.\left.f_{d} L_{1} T_{r}\right)\right]^{T}$ are the Doppler steering vectors.

The steering vector for the vectorial case [6] can be derived by the kronecker product of these vectors and by setting $L_{1}=L$ or $L_{2}=L$. The white noise $\operatorname{vec}(\mathcal{N})$ and the clutter noise $\operatorname{vec}(\mathbf{C})$ follows respectively the distributions $\mathcal{C N}\left(\mathbf{0}, \sigma^{2} \mathbf{I}\right)$ and $\mathcal{C N}\left(\mathbf{0}, \mathbf{R}_{c}\right)$. Let us denote by $\mathbf{R}=\mathbf{R}+\sigma^{2} \mathbf{I}$ the total covariance matrix. From the covariance matrix $\mathbf{R}$, we can define the covariance tensor $\mathcal{R} \in \mathbb{C}^{N \times M \times L_{1} \times L_{2} \times N \times M \times L_{1} \times L_{2}}:$

$$
\mathcal{R}=S q M a t^{-1}(\mathbf{R})
$$

\section{B. Tensorial LR-STAP MIMO Filters}

1) STAP Filter: The partition in this configuration is $\mathcal{A}=N, M, L_{1}, L_{2}$. Let $\mathcal{A}_{1}, \ldots, \mathcal{A}_{Q}$ be a partition of $\mathcal{A}=N, M, L_{1}, L_{2}$. At this moment, we do not consider a specific partition and the choice will be discussed in the next subsection.

For the given partition, the optimal tensor filter, which maximizes the SINR output is given by:

$$
\mathcal{W}_{\text {opt }}=\operatorname{vec}^{-1}\left(\operatorname{SqMat}(\mathcal{R})^{-1} \operatorname{vec}(\boldsymbol{S})\right) .
$$

It is shown in [16], [17] that the filter $\mathcal{W}_{\text {opt }}$ is equivalent to the optimal vectorial filter. Therefore, the tensor modelling is here unnecessary.

On the other hand, it has been shown [16], [17] that the LR tensor filter based on AU-HOSVD for a given partition $\mathbb{A}_{1}, \ldots, \mathbb{A}_{Q}$ can bring better results than the LR vectorial one. Its expression depends on the steering tensor and the LR approximation of the covariance tensor for the given partition (see Eq. (8)):

$$
\mathcal{W}_{l r\left(\mathbb{A}_{1}, \ldots, \mathbb{A}_{P}\right)}=\mathcal{S} \times_{\mathbb{A}_{1}} \mathbf{U}_{0}^{\left(\mathbb{A}_{1}\right)} \mathbf{U}_{0}^{\left(\mathbb{A}_{1}\right) H} \ldots \times_{\mathbb{A}_{P}} \mathbf{U}_{0}^{\left(\mathbb{A}_{P}\right)} \mathbf{U}_{0}^{\left(\mathbb{A}_{P}\right) H}
$$




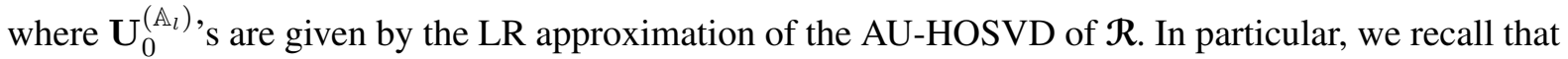
$\mathbf{U}_{0}^{\left(\mathbb{A}_{p}\right)}=\left[\mathbf{u}_{r_{\mathbb{A}_{p}}+1}^{\left(\mathbb{A}_{p}\right)} \ldots \mathbf{u}_{\mathbb{A}_{p}}^{\left(\mathbb{A}_{p}\right)}\right]$ where $r_{\mathbb{A}_{p}}$ is the multimode rank for the unfolding $\mathbb{A}_{p}$. The demonstration of its derivation has been shown in [16]. The output of the filter is given by:

$$
y=\left|<\mathcal{W}_{\mathbb{A}_{1}, \ldots, \mathbb{A}_{P}}, \boldsymbol{x}>\right|
$$

As the tensor covariance is unknown, it has to be estimated from the secondary data $X_{k}$ in order to obtain the Sample Covariance Tensor $\hat{\mathcal{R}}[18]$ :

$$
\hat{\mathcal{R}}=\frac{1}{K} \sum_{k=1}^{K} \boldsymbol{x}_{k} \circ \boldsymbol{X}_{k}^{*}
$$

The AU-HOSVD and the LR approximation of $\hat{\mathcal{R}}$ allow to obtain the matrices $\hat{\mathbf{U}}_{0}^{\left(\mathbb{A}_{1}\right)}, \ldots, \hat{\mathbf{U}}_{0}^{\left(\mathbb{A}_{P}\right)}$. By plugging these matrices in Eq. (17) at the places of the matrices $\mathbf{U}_{0}^{\left(\mathbb{A}_{1}\right)}, \ldots, \mathbf{U}_{0}^{\left(\mathbb{A}_{P}\right)}$, we obtain the tensorial adaptive LR filter $\hat{\mathcal{W}}_{\operatorname{lr}\left(\mathbb{A}_{1}, \ldots, \mathbb{A}_{P}\right)}$. We notice that it depends on the choice of the partition and the evaluation of the corresponding multimode ranks.

This choice could be helped by the evaluation of the adaptive filter performance. We then investigate the SINR loss for the tensorial case. In the case of $\hat{\mathcal{W}}_{l r\left(\mathbb{A}_{1}, \ldots, \mathbb{A}_{P}\right)}$ (denoted $\hat{\mathcal{W}}_{l r}$ in the equation for more clarity), the SINR loss expression is:

$$
\rho=\frac{\left|\left(\operatorname{vec}\left(\hat{\mathcal{W}}_{l r}\right)^{H} \operatorname{vec}(\boldsymbol{S})\right)\right|^{2}}{\operatorname{vec}\left(\hat{\mathcal{W}}_{l r}\right)^{H} \operatorname{SqMat}(\boldsymbol{\mathcal { R }}) \operatorname{vec}\left(\hat{\mathcal{W}}_{l r}\right) \operatorname{vec}(\boldsymbol{S})^{H} \operatorname{SqMat}(\boldsymbol{\mathcal { R }})^{-1} \operatorname{vec}(\boldsymbol{S})}
$$

As stated at the beginning of the current section, it could be really interesting to theoretically derive the SINR loss in particular for the partition choice. In [29], thanks to perturbation analysis, it has been proposed a theoretical study of a Tensor MUSIC algorithm built from the HOSVD but the problem is easier than for the SINR loss. Indeed, the SINR loss is a quantity more complicated than the MUSIC criterion and the AU-HOSVD is also more complex than the HOSVD. Nevertheless, by inspecting the results of the SINR loss for vectorial LR filters of Eq. (12), we guess that the SINR loss of tensorial LR filters will depend on the multimode ranks of the clutter contribution. Since these ranks could be different for each partition, their study is required for a correct choice of the partition. Moreover, in the next subsection, it will be shown that the multimode ranks for all partitions could be evaluated theoretically which will be helpful for the choice of the partition and especially for the computation of the tensorial LR filter of Eq. (17).

2) Partitions: A representation of the tensor model and some of its corresponding partitions are represented in Figure 2.

The different choices of partition for the tensor model are also presented in table I. The two first partitions, vector and HOSVD, can be seen as particular case of the AU-HOSVD.

To limit the number of studied filters, only the most interesting ones will be considered. As stated on previous subsection III-A, only filters which combine both features $F_{1}$ and $F_{2}$ (ST and VA) are 


\begin{tabular}{|c|l|c|c||}
\hline \hline Partition & Filters & Methods & Unfolding features \\
\hline $\mathbb{A}_{1}=\{1,2,3,4\}$ & $\hat{\mathcal{W}}_{l r(1,2,3,4)}=\hat{\mathbf{w}}_{L R}$ & Vector & ST,VA \\
\hline $\mathbb{A}_{1}=\{1\}, \mathbb{A}_{2}=\{2\}, \mathbb{A}_{3}=\{3\}, \mathbb{A}_{4}=\{4\}$ & $\hat{\mathcal{W}}_{l r(1 / 2 / 3 / 4)}$ & HOSVD & Rx / Tx / Pulse $T_{r} /$ Pulse $T_{r}^{\prime}$ \\
\hline $\mathbb{A}_{1}=\{1\}, \mathbb{A}_{2}=\{2,3,4\}$ & $\hat{\mathcal{W}}_{l r(1 / 2,3,4)}$ & AU-HOSVD & Rx / ST \\
\hline $\mathbb{A}_{1}=\{2\}, \mathbb{A}_{2}=\{1,3,4\}$ & $\hat{\mathcal{W}}_{l r(2 / 1,3,4)}$ & AU-HOSVD & Tx / ST \\
\hline $\mathbb{A}_{1}=\{3\}, \mathbb{A}_{2}=\{1,2,4\}$ & $\hat{\mathcal{W}}_{l r(3 / 1,2,4)}$ & AU-HOSVD & Pulse $T_{r} / \mathrm{ST}$, VA \\
\hline $\mathbb{A}_{1}=\{4\}, \mathbb{A}_{2}=\{1,2,3\}$ & $\hat{\mathcal{W}}_{l r(4 / 1,2,3)}$ & AU-HOSVD & Pulse $T_{r}^{\prime} /$ ST, VA \\
\hline $\mathbb{A}_{1}=\{1,2\}, \mathbb{A}_{2}=\{3,4\}$ & $\hat{\mathcal{W}}_{l r(1,2 / 3,4)}$ & AU-HOSVD & VA / Pulse $T_{r}$, Pulse $T_{r}^{\prime}$ \\
\hline $\mathbb{A}_{1}=\{1,3\}, \mathbb{A}_{2}=\{2,4\}$ & $\hat{\mathcal{W}}_{l r(1,3 / 2,4)}$ & AU-HOSVD & ST / ST \\
\hline $\mathbb{A}_{1}=\{1,4\}, \mathbb{A}_{2}=\{2,3\}$ & $\hat{\mathcal{W}}_{l r(1,4 / 2,3)}$ & AU-HOSVD & ST / ST \\
\hline $\mathbb{A}_{1}=\{1,2\}, \mathbb{A}_{2}=\{3\}, \mathbb{A}_{3}=\{4\}$ & $\hat{\mathcal{W}}_{l r(1,2 / 3 / 4)}$ & AU-HOSVD & VA / Pulse $T_{r} /$ Pulse $T_{r}^{\prime}$ \\
\hline $\mathbb{A}_{1}=\{1,3\}, \mathbb{A}_{2}=\{2\}, \mathbb{A}_{3}=\{4\}$ & $\hat{\mathcal{W}}_{l r(1,3 / 2 / 4)}$ & AU-HOSVD & ST / Tx / Pulse $T_{r}^{\prime}$ \\
\hline $\mathbb{A}_{1}=\{1,4\}, \mathbb{A}_{2}=\{2\}, \mathbb{A}_{3}=\{3\}$ & $\hat{\mathcal{W}}_{l r(1,4 / 2 / 3)}$ & AU-HOSVD & ST / Tx / Pulse $T_{r}$ \\
\hline $\mathbb{A}_{1}=\{2,3\}, \mathbb{A}_{2}=\{1\}, \mathbb{A}_{3}=\{4\}$ & $\hat{\mathcal{W}}_{l r(2,3 / 1 / 4)}$ & AU-HOSVD & ST / Rx / Pulse $T_{r}^{\prime}$ \\
\hline $\mathbb{A}_{1}=\{2,4\}, \mathbb{A}_{2}=\{1\}, \mathbb{A}_{3}=\{3\}$ & $\hat{\mathcal{W}}_{l r(2,4 / 1 / 3)}$ & AU-HOSVD & ST / Tx / Pulse $T_{r}$ \\
\hline $\mathbb{A}_{1}=\{3,4\}, \mathbb{A}_{2}=\{1\}, \mathbb{A}_{3}=\{2\}$ & $\hat{\mathcal{W}}_{l r(3,4 / 1 / 2)}$ & AU-HOSVD & Pulse $T_{r}$, Pulse $T_{r}^{\prime} /$ Rx / Tx \\
\hline \hline
\end{tabular}

TABLE I

DESCRIPTION OF THE LR FILTERS PROVIDED BY AU-HOSVD FOR THE TENSORIAL MODEL OF MIMO-STAP. THE MOST INTERESTING LR FILTERS ARE HIGHLIGHTED IN YELLOW.

interesting for MIMO-STAP. By inspecting table I, we conclude that two partitions possess both these features:

- Partition $\left(\mathbb{A}_{1}=\{4\}, \mathbb{A}_{2}=\{1,2,3\}\right)$. Transmit and receive spatial dimensions and reduced Doppler dimension are combined. Each subset of pulses are treated separately. $\mathbb{A}_{2}=\{1,2,3\}$ corresponds to a MIMO radar with a reduced number of pulses, then the corresponding multimode ranks are computed (the details are in Appendix B):

$$
\begin{aligned}
& r_{1,2,3}=N+\gamma(M-1)+\beta\left(L_{1}-1\right) \\
& r_{4}=L_{1} \beta\left(L_{2}-1\right)+1
\end{aligned}
$$

- Partition $\mathbb{A}_{1}=\{3\}, \mathbb{A}_{2}=\{1,2,4\}$. Transmit and receive spatial dimensions and pulse subsets with higher $T_{r}$ are combined. The reduced pulse dimension is treated separately. $\mathbb{A}_{2}=\{1,2,4\}$ corresponds to a MIMO radar with reduced number of pulses spaced by $T_{r}^{\prime}=L_{1} T_{r}$. The multimode ranks are derived as follows (the details are in Appendix B):

$$
\begin{aligned}
& r_{1,2,4}=N+\gamma(M-1)+L_{1} \beta\left(L_{2}-1\right) \\
& r_{3}=\beta\left(L_{1}-1\right)+1
\end{aligned}
$$

The main drawback with this partition is the increase of the pulse repetition interval $T_{r}^{\prime}$ which 
leads to the reduction of the non-ambiguous Doppler domain for clutter suppression. Another way to see it is to consider the increase of the parameter $\beta^{\prime}=L_{1} \beta$ which introduces ambiguity in Doppler [2][19].

Naturally, it seems that the first partition is the most interesting. But, it remains important to study the multimode ranks $r_{1,2,3}, r_{4}, r_{1,2,4}$ and $r_{3}$ as a function of the different parameters $M, N, L_{1}$ and $L_{2}=L / L_{1}$. We notice that the choice of $L_{1}$ will affect the rank values. This study is important to respect the feature $F_{3}$.

\section{NUMERICAL RESULTS}

\section{A. Configuration}

We consider a colocated MIMO radar (and then coherent) with $M=4$ transmitters, $N=4$ receivers and $L=64$ pulses. The MIMO radar is in side-looking configuration. We simulate a ground clutter using Ward model [19] with $\beta=1$. We assume that the waveforms are ideally orthogonal and can then be perfectly disentangle at each receiver. At last, we consider MIMO sparse configuration with $d r=\lambda_{0} / 2$ and $d e=N d r$. Sparse MIMO allows the generation of the longest virtual antenna array [7] and thus gives higher angular resolution compared to SIMO.

\section{B. Ranks}

Figure 3 presents the vectorial and the multimode ranks $\left(r_{1,2,3}, r_{4}, r_{1,2,4}\right.$ and $\left.r_{3}\right)$ of the clutter contribution as a function of $L_{1}$. First, we notice that the multimode ranks have smaller values than the vectorial one. We could expect in this case better results of tensorial adaptive LR filters.

For the partition $\left(\mathbb{A}_{1}=\{4\}, \mathbb{A}_{2}=\{1,2,3\}\right)$, we notice that a small value of $L_{1}$ seems to be promising to have two low multimode ranks. On the contrary a small value of $L_{1}$ for the partition $\left(\mathbb{A}_{1}=\{3\}, \mathbb{A}_{2}=\{1,2,4\}\right)$ leads to a high multimode rank $r_{3}$. A mean value of $L_{1}$ for this last partition will be more suitable in order to have low multimode ranks.

\section{MIMO-STAP Performances}

We present in this section the SINR loss of the LR tensorial filter. Since, it is really difficult to estimate theoretically the SINR loss distribution of Eq. (20), we decide to evaluate it by means of a Monte Carlo simulation with 1000 trials. First, we study the SINR losses of different MIMO-STAP filters as of the normalized Doppler frequency. The number of secondary data is set to $K=158$ which is equal to twice the rank $r=79$ of the clutter in the vectorial case. The tensorial LR filters will be compared to the vectorial LR filter and the Pre-Doppler algorithm. In this last algorithm, the size of the sub-pulse $K_{\text {sub }}$ is set to 2 as generally proposed in the literature [19]. 
We present in Figure 4, the SINR losses of the tensorial LR filter with the first partition $\{1,2,3 / 4\}$, the vectorial LR filter and the Pre-Doppler as function of the normalized Doppler frequency. The number of reduced pulses $L_{1}$ is set to three values $8,16,32$. Since $K=2 r$, we have the well-known 3dB loss for the LR vectorial case [10]. As expected for high Doppler frequencies, the Pre-Doppler algorithm gives better results than the vectorial LR filter. The tensorial LR filters allows better SINR losses with values around $-1 \mathrm{~dB}$ compared to the $\mathrm{LR}$ vectorial one. Compared to Pre-Doppler, the MIMO angular resolution is preserved for the LR tensorial filters. The LR tensorial filters almost give slight different results for different values of $L_{1}$. For $L_{1}=8$, we achieve around $0.5 \mathrm{~dB}$ SINR loss with a slightly wider clutter notch compared to the cases of $L_{1}=16$ and $L_{1}=32$. For $L_{1}=32$, the SINR loss is around $-1 \mathrm{~dB}$ with a clutter notch as narrow as for LR vectorial one. For the case $L_{1}=16$, LR tensorial filter gives results in between the two others LR tensorial filters. For large Doppler, this analysis of the SINR loss agrees with the one of the multimode ranks in the previous subsection.

We present now in Figure 5 the SINR losses as function of the normalized Doppler frequency for the partition $\{1,2,4 / 3\}$. We evaluate two values of $L_{1}$. When the pulse repetition interval is increased by $L_{1}=2$ i.e. the non-ambiguous Doppler is reduced by 2 compared to the vectorial case, then $L_{2}=32$. In the same way for $L_{2}=16$, the non-ambiguous is further reduced by $L_{1}=4$. The results clearly illustrate the reduction of the non-ambiguous Doppler domain. Thus, we achieve SINR losses of $-2 \mathrm{~dB}$ for $L_{1}=2$ and $-1 \mathrm{~dB}$ for $L_{1}=4$. This last result is similar to the analysis of the rank of the previous subsection where a small value $L_{1}$ is less suitable than a large value. But with this partition, the choice of $L_{1}$ also affects the number of ambiguities. In this case, a large value of $L_{1}$ is not advised. Therefore, a compromise between small SINR loss and ambiguities has to be done with this partition.

Figure 6 shows the convergence of the SINR loss in terms of the number of secondary data. The speed is set to $v=-7 \mathrm{~m} / \mathrm{s}$ to evaluate the MIMO-STAP performance for a target close to the clutter ridge. We only present the results for the LR tensorial filters associated to the best partitions: we naturally choose the LR filter $\{1,2,3 / 4\}$ with $L_{1}=16$ (good compromise between the notch clutter and the SINR loss). We clearly see that the tensorial adaptive LR filter built from the AU-HOSVD greatly outperforms the two vectorial STAP approaches. The minimal SINR loss is achieved with a very fast convergence.

\section{NUMERICAL RESULTS ON A REALISTIC CONFIGURATION}

\section{A. Configuration}

The radar configuration is the same as in the previous section. We consider a MIMO radar in sparse configuration. We simulate a ground clutter with Gaussian distribution and a $C N R=40 \mathrm{~dB}$. 
The target is a slow target located at $v=5 \mathrm{~m} / \mathrm{s}$ and at an angle $\phi=0^{\circ}$ i.e. near the clutter. Moreover the target range is $R=3.75 \mathrm{~km}$.

The MIMO waveforms are slow-time coded waveforms, specifically DDMA (Doppler Division Multiple Access) waveforms [30][31]. This waveform consists in a coding sequence applied on pulses and a common waveform in fast-time. The common waveform in fast-time is a LFM (Linear Frequency Modulation) chirp with a central frequency $f_{0}=450 \mathrm{MHz}$, a bandwidth $B=4 \mathrm{MHz}$ and a duration $t_{p}=10^{-4} \mathrm{~s}$. The DDMA coding generates transmit signals in different Doppler domains. Thus DDMA waveforms are orthogonal in their respective Doppler domain. The drawbacks of the DDMA waveforms are the limitation of the non-ambiguous Doppler domain and the ambiguities due to the response of the clutter in the reduced non-ambiguous Doppler domain. Nevertheless, as the coding is performed in slow-time, the MIMO data are still i.i.d. in range which allows to use conventional STAP approach.

Figure 7 shows the range/Doppler map of the simulated scene. Since the radar MIMO emits DDMA waveforms, the clutter response is repeated in the Doppler space. Another way to see it is to say that the non-ambiguous Doppler is reduced from $[-5050] \mathrm{m} / \mathrm{s}$ to $M$ times less, i.e. $[-12.512 .5] \mathrm{m} / \mathrm{s}$. The target is not visible because its SNR is way below the CNR.

We present in Figure 8 the angle/Doppler map at the target range. The clutter has a response consisting in diagonal ridges in the angle/Doppler space. The main ridge is the direct response of the clutter whereas the others ridges are due to DDMA waveform ambiguities.

\section{B. STAP filtering results}

We perform STAP filtering on the simulated data at the target range. We use $2 r=158$ secondary data, free of target response and target sidelobes. Figure 9 shows the angle/Doppler map after LR vectorial filtering. The clutter is reduced and the target and its ambiguous responses are clearly visible. This result illustrates the exploitation of the LR property of the clutter to suppress it since only 158 secondary are used compared to the fully adaptive STAP which requires $2 M N L=2048$ secondary data. We present in Figure 10 the angle/Doppler map after the proposed tensorial adaptive LR filter using the partition $\{1,2,3 / 4\}$ with $L_{1}=16$. We clearly see the improvement of the clutter reduction compared to the LR filter. Moreover, the clutter notch resolution is preserved which allows the detection of the slow target.

\section{CONCLUSION}

We have developed in this paper a new adaptive LR filter for MIMO-STAP application based on a tensorial modelling of the data. This filter is based on an extension of the HOSVD (which is also one possible extension of SVD to the tensor case), called AU-HOSVD, which allows to consider the 
combinations of dimensions. This property is necessary to keep the advantages of the STAP and the MIMO characteristics of the data. Since the tensorial LR filter depends on a partition of the data, we showed that the choice of the good partition (as well as the tensorial modelling) had to follow 3 rules:

- Space-Time (ST) combination: STAP processing is based on the combination of space and time dimensions.

- Virtual Antenna (VA): in coherent MIMO, resolution is improved through the combination of the transmit and receive arrays that creates a virtual antenna.

- Low Rank: LR property of the clutter covariance tensor is essential to derive efficient LR filter. It is important to have the ranks the smallest in particular with respect of the total size of the data.

With these features, we noticed that only two partitions (and so two LR filters) had been suitable for MIMO-STAP. We also derived the theoretical formulation of multimode ranks for all partitions which is necessary to compute LR methods. Results on simulated data show the good performance of the AU-HOSVD LR filters in terms of secondary data and clutter notch. Finally results on simulated using slow-time coded waveforms show the robustness of the approach for practical configuration.

In future work, the SINR loss distribution will be theoretically investigated based on the work of [26][29].

\section{APPENDIX}

\section{A. Clutter rank derivation}

Clutter rank derivation have been proposed by Brennnan in [21] for phased array in ULA and sidelooking configuration and for $\lambda_{0} / 2$ spaced elements. Its extension to MIMO radar in same configuration for perfectly orthogonal waveforms has been proposed in [6]. We propose here the derivation of the clutter ranks corresponding to the different partitions. The ranks are derived using general formulation proposed by [22], [23].

The elements of the clutter response can be written as:

$$
y_{n, m, l}=\sum_{i=0}^{N_{c}} \alpha_{i} e^{j 2 \pi f_{s, i}(n+\gamma m+\beta l)}
$$

where $N_{c}$ is the number of clutter patches, $f_{s, i}$ is the spatial frequency for the $i$-th clutter patch and $\alpha_{i}$ is the amplitude of the $i$-th clutter patch. As we assume that $d_{r}=\lambda_{0} / 2$, we have $-0.5<f_{s, i}<0.5$. The vector of the clutter response $\mathbf{y} \in \mathbb{C}^{N M L \times 1}$ can be written as:

$$
\mathbf{y}=\sum_{i=0}^{N_{c}} \alpha_{i} \mathbf{c}_{i}
$$


where $c_{i}=e^{j 2 \pi f_{s, i} n+\gamma m+\beta l}$. The clutter covariance matrix is then written as:

$$
\mathbf{R}_{c}=\sum_{i=0}^{N_{c}} E\left[\alpha_{i} \alpha_{i}^{*}\right] \mathbf{c}_{i} \mathbf{c}_{i}^{H} .
$$

Using Eq. (25) we conclude than $\operatorname{rank}\left(\mathbf{R}_{c}\right)=\operatorname{span}(\mathbf{C})$ with $\mathbf{C}=\left(\begin{array}{llll}c_{1} & c_{2} & \ldots & c_{N_{c}}\end{array}\right)$. The elements of the clutter response $\mathbf{c}_{i}$ can be rewritten by using a space-time sampling as follows:

$$
c_{i, p}=e^{j 2 \pi f_{s, i} x_{p}}
$$

where $0 \leq p<N M L$ is the space-time sampling index number and $x_{p}=n+\gamma m+\beta l$. The span of $\mathbf{C}$ is then the number of distinct values taken by $x_{p}$. The elements $x_{p}$ can be written as:

$$
x_{p}=k_{1} h_{1}+k_{2} h_{2}
$$

where $k_{1}=1, h_{1}=n+\gamma m, k_{2}=\beta$ and $h_{2}=l$. It is shown in [22] that the rank of the clutter covariance matrix is derived as:

$$
r= \begin{cases}H_{1} H_{2} & \text { for } H_{1} \leq k_{2} \text { or } H_{2} \leq k_{1} \\ H_{1} k_{1}+H_{2} k_{2}-k_{1} k_{2} & \end{cases}
$$

where $H_{1}$ and $H_{2}$ are the total numbers of elements for the respective dimensions of the data and $k_{1}$ and $k_{2}$ are the parameters associated to the spatial frequency $f_{s}$. The complete demonstration of Eq. (28) can be found in [22]. First equation corresponds to the full rank case. Second equation corresponds to the deficient rank case. Rank formulation given by Eq. (28) has been developed for SIMO radar by considering 2 dimensions. This formulation can also be used for MIMO radar with more than 2 dimensions.

\section{B. Ranks for the partitions of the tensor model}

- Partition $\mathbb{A}=\{1,2,3,4\}$. This case corresponds to the vectorial case. Then its corresponding rank is given by:

$$
r_{1,2,3}=\left\{\begin{array}{l}
(N+\gamma(M-1)) L \\
\quad \text { or } N+\gamma(M-1) \leq \beta \text { or } L \leq 1 \\
N+\gamma(M-1)+\beta(L-1)
\end{array}\right.
$$

The condition $N+\gamma(M-1) \leq \beta$ is rarely obtained and $L \leq 1$ does not make sense for pulse radar. It is that why that he Generalized Brennan's rule [6] only considers the second formulation for the deficient rank.

- Partitions $\mathbb{A}_{1}=\{1\}, \mathbb{A}_{2}=\{2\}, \mathbb{A}_{3}=\{3\}, \mathbb{A}_{4}=\{4\}$. In this case, each partition contains only one single dimension. Eq. (28) reduces in this case as:

$$
r=\left\{\begin{array}{lll}
H_{1} & \text { for } H_{1}=1 \text { or } 1 \leq k_{1} \\
k_{1}\left(H_{1}-1\right)+1 &
\end{array}\right.
$$


We obtain then the following ranks:

$$
r_{1}=N
$$

$$
\begin{aligned}
& r_{2}= \begin{cases}M & \text { for } M=1 \text { or } 1 \leq \gamma \\
\gamma(M-1)+1,\end{cases} \\
& r_{3}=\left\{\begin{array}{l}
L_{1} \\
\beta\left(L_{1}-1\right)+1
\end{array} \text { for } L_{1}=1 \text { or } 1 \leq \beta\right.
\end{aligned}
$$

and,

$$
r_{4}= \begin{cases}L_{2} & \text { for } L_{2}=1 \text { or } 1 \leq \beta^{\prime} \\ \beta^{\prime}\left(L_{2}-1\right)+1 & \end{cases}
$$

with $\beta^{\prime}=2 v_{a} T_{r} L_{1} / d_{r}=L_{1} \beta$.

- Partition $\mathbb{A}=\{2,3,4\}$.

$$
r_{2,3,4}= \begin{cases}M L & \text { for } M \leq \beta \text { or } L \leq \gamma \\ \gamma M+\beta L-\beta \gamma & \end{cases}
$$

- Partition $\mathbb{A}=\{1,3,4\}$.

$$
r_{1,3,4}= \begin{cases}N L & \text { for } N \leq \beta \text { or } L \leq 1 \\ N+\beta(L-1) & \end{cases}
$$

In the rank deficient case, we find the Brennan's rule [21].

- Partition $\mathbb{A}=\{1,2,4\}$. In that case we consider a MIMO system with a few pulse with a increased PRI $T_{r}^{\prime}=L_{1} T_{r}$.

$$
r_{1,2,4}=\left\{\begin{array}{l}
(N+\gamma(M-1)) L_{2} \\
\quad \text { for }(N+\gamma(M-1)) \leq L_{1} \beta \quad \text { or } \quad L_{2}=1 \\
N+\gamma(M-1)+L_{1} \beta\left(L_{2}-1\right)
\end{array}\right.
$$

This is the Generalized Brennan's rule for $\beta^{\prime}=L_{1} \beta$ and for number of pulses $L_{2}$.

- Partition $\mathbb{A}=\{1,2,3\}$.

$$
r_{1,2,3}=\left\{\begin{array}{l}
(N+\gamma(M-1)) L_{1} \\
\quad \text { for }(N+\gamma(M-1)) \leq \beta \quad \text { or } \quad L_{1}=1 \\
N+\gamma(M-1)+\beta\left(L_{1}-1\right)
\end{array}\right.
$$

It is the Generalized Brennan's rule for a number of pulses $L_{1}$.

- Partition $\mathbb{A}=\{1,2\}$. In this case, using Eq. (28) the rank $r_{1,2}$ is given by:

$$
r_{1,2}= \begin{cases}N M & \text { for } N \leq \gamma \text { or } M \leq 1 \\ N+\gamma(M-1) & \end{cases}
$$


$r_{1,2}$ can also be seen as the number of phase centers of the MIMO virtual antenna [32].

- Partition $\mathbb{A}=\{1,3\}$. This partition associates the $N$ receivers with the $L_{1}$ reduced pulses. The rank is derived as:

$$
r_{1,3}= \begin{cases}N L_{1} & \text { for } N \leq \beta \text { or } L_{1} \leq 1 \\ N+\beta\left(L_{1}-1\right) & \end{cases}
$$

- Partition $\mathbb{A}=\{1,4\}$. This partition associates the $N$ receivers with the $L_{2}$ pulses spaced by $T_{r}^{\prime}$. The rank is given as:

$$
r_{1,3}= \begin{cases}N L_{2} & \text { for } N \leq \beta \text { or } L_{2} \leq 1 \\ N+\beta^{\prime}\left(L_{2}-1\right) & \end{cases}
$$

- Partition $\mathbb{A}=\{2,3\}$. This partition associates the $M$ transmitters with the $L_{1}$ pulses. The rank is given as:

$$
r_{2,3}= \begin{cases}M L_{1} & \text { for } M \leq \beta \text { or } L_{1} \leq \gamma \\ \gamma M+\beta L_{1}-\beta \gamma & \end{cases}
$$

- Partition $\mathbb{A}=\{2,4\}$. This partition associates the $M$ transmitters with the $L_{2}$ pulses spaced by $T_{r}^{\prime}$. The rank is derived as:

$$
r_{2,4}= \begin{cases}M L_{2} & \text { for } M \leq \beta \text { or } L_{2} \leq \gamma \\ \gamma M+\beta L_{2}-\beta^{\prime} \gamma & \end{cases}
$$

- Partition $\mathbb{A}=\{3,4\}$. In this partition we consider all the pulses. Then the rank is equal to:

$$
r_{3,4}= \begin{cases}L & \text { for } \beta \geq 1 \text { or } L_{2} \leq \beta \\ \beta(L-1)+1 & \end{cases}
$$

\section{REFERENCES}

[1] J. Ward, Space-Time Adaptive Processing for Airborne Radar, Lincoln Laboratoy, MIT, Lexington, Massachusetts, USA, 1994.

[2] R. Klemm, Principles of Space-Time Adaptive Processing, IEE Press, London, U.K., 2002.

[3] E. Fishler, A. Haimovivh, R. Blum, L. Cimini, D. Chizhik, and R. Valenzuela, "MIMO radar: an idea whose time has come," in Proceedings of IEEE International Radar Conference, April 2004, pp. 71-78.

[4] D.W. Bliss and K.W. Forsythe, "Multiple-input multiple-output (MIMO) radar and imaging: degrees of freedom and resolution," in Conference Record of the Thirty-Seventh Asilomar Conference on Signals, Systems and Computers, 2003., November 2003, vol. 1, pp. 54 - 59 Vol.1.

[5] J. Li and P. Stoica, "MIMO radar with colocated antennas," IEEE Signal Processing Magazine, vol. 24, no. 5, pp. 106 - 114, September 2007.

[6] C.Y. Chen and P.P. Vaidyanathan, "MIMO radar space-time adaptive processing using prolate spheroidal wave functions," IEEE Transactions on Signal Processing, vol. 56, no. 2, pp. 623-635, February 2008.

[7] J. Li and P. Stoica, MIMO Radar Signal Processing, Wiley, 1st edition, 2009.

[8] I.P. Kirsteins and D. Tufts, "Adaptive detection using a low rank approximation to a data matrix," IEEE Transactions on Aerospace and Electronic System, vol. 30, pp. 55 - 67, 1994. 
[9] A. Haimovich, "The eigencanceler: Adaptive radar by eigenanalysis methods," IEEE Transactions on Aerospace Electronic Systems, vol. 32, no. 2, pp. 532 - 542, April 1996.

[10] A.M. Haimovich and M. Berin, "Eigenanalysis-based space-time adaptive radar: performance analysis," Aerospace and Electronic Systems, IEEE Transactions on, vol. 33, no. 4, pp. 1170-1179, Oct 1997.

[11] C.D. Peckham, A.M. Haimovich, T.F. Ayoub, J.S. Goldstein, and I.S. Reed, "Reduced-rank STAP performance analysis," IEEE Transactions on Aerospace and Electronic Systems, vol. 36, no. 2, pp. 664 - 676, April 2000.

[12] T. Kolda and B. Bader, "Tensor decompositions and applications," SIAM Review, vol. 51, pp. 455 - 500, 2009.

[13] L. De Lathauwer, B. De Moor, and J. Vandewalle, “A multilinear singular value decomposition," SIAM J. Matrix Anal. Apl., vol. 24, no. 4, pp. 1253-1278, 2000.

[14] R.A. Harshman, "Foundations of the PARAFAC procedure: Models and conditions for an 'explanatory' multi-modal analysis," UCLA Working Papers in Phonetics, vol. 16, pp. 1-84, 1970.

[15] L.R. Tucker, "Some mathematical notes on three-mode factor analysis," Psychometrika, vol. 31, pp. 279-311, 1966.

[16] M. Boizard, G. Ginolhac, F. Pascal, and P. Forster, "Low-rank filter and detector for multidimensional data based on an alternative unfolding HOSVD. application to polarimetric STAP," in EURASIP Journal on Advances in Signal Processing, 2014.

[17] M. Boizard, G. Ginolhac, F. Pascal, and P. Forster, "A new tool for multidimensional low-rank STAP filter: Cross HOSVDs," in Proceedings of EUSIPCO, Bucharest, Romania, 2012.

[18] M. Haardt, F. Roemer, and G. Del Galdo, "Higher-order SVD-based subspace estimation to improve the parameter estimation accuracy in multidimensionnal harmonic retrieval problems," IEEE Trans. on Proc. Sig. Proc., vol. 56, no. 7, pp. 3198-3213, July 2008.

[19] J. Ward, Space-time adaptive processing for airborne radar, Technical Report 1015, Massachusetts Institute of Technology, Lexington, MA, December 1994.

[20] E.J. Baranoski, "Improved pre-doppler stap algorithm for adaptive clutter nulling in airborne radars," in Twenty-Ninth Asilomar Conference on Signals, Systems and Computers, October 1995, vol. 2, pp. 1173 -1177.

[21] L.E. Brennan and I.S. Reed, “Theory of adaptive radar," IEEE Transactions on Aerospace and Electronic Systems, vol. AES-9, no. 2, pp. 237 - 252, March 1973.

[22] Yong Wu, Jun Tang, and Yingning Peng, "Clutter rank of sparse linear array radar," in International Conference on Radar, 2006. CIE '06., 2006, pp. 1 - 4.

[23] N.A. Goodman and J. M. Stiles, “On clutter rank observed by arbitrary arrays," IEEE Transactions on Signal Processing, vol. 55, no. 1, pp. 178-186, January 2007.

[24] L. De Lathauwer, B. De Moor, and J. Vandewalle, "On the best rank-1 and rank- $\left(r_{1}, r_{2}, \ldots, r_{n}\right)$ approximation and applications of higher-order tensors," SIAM J. Matrix Anal. Apl., vol. 21, no. 4, pp. 1324-1342, 2000.

[25] D.W. Bliss, K.W. Forsythe, S.K. Davis, G.S. Fawcett, D.J. Rabideau, L.L. Horowitz, and S. Kraut, "GMTI MIMO radar," in International Waveform Diversity and Design Conference 2009, February 2009, pp. 118 -122.

[26] G. Ginolhac, P. Forster, F. Pascal, and J.P. Ovarlez, "Performance of two low-rank STAP filters in a heterogeneous noise,” IEEE Trans. Signal Process., vol. 61, no. 1, pp. 57 - 61, January 2013.

[27] H. Wang and L. Cai, "On adaptive spatial-temporal processing for airborne surveillance radar systems," IEEE Trans. on Aerospace and Electronic Systems, vol. 30, no. 3, pp. 660 - 670, July 1994.

[28] R.C. Di Pietro, "Extended factored space-time processing for airborne radar systems," in Proceedings of 26th Asilomar Conference on Signal, Systems, and Computers, October 1992, vol. 1, pp. 425-430.

[29] P. Forster and G. Ginolhac, "Derivation of the theoretical performances of a tensorial MUSIC algorithm," Submitted in Signal Processing, 2015.

[30] V.F. Mecca, D. Ramakrishnan, and J.L. Krolik, "MIMO radar space-time adaptive processing for multipath clutter mitigation," in Fourth IEEE Workshop on Sensor Array and Multichannel Processing, 2006, July 2006, pp. $249-253$. 
[31] V.F. Mecca and J.L. Krolik, "MIMO STAP clutter mitigation performance demonstration using acoustic arrays," in 42nd Asilomar Conference on Signals, Systems and Computers, 2008, October 2008, pp. 634-638.

[32] J. Li and P. Stoica, MIMO radar - Diversity means superiority, J. Li and P. Stoica, Eds, New York: Wiley, 2009. 


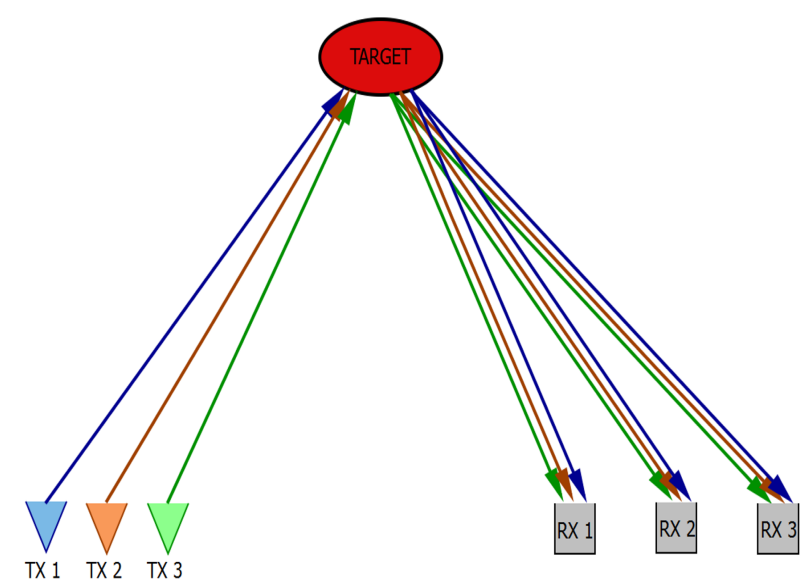

Fig. 1. MIMO radar in coherent configuration.

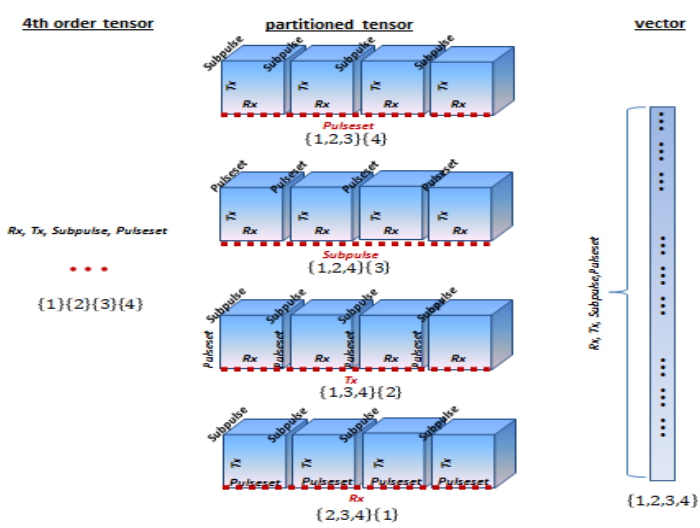

Fig. 2. Tensor model and its corresponding last partitions.

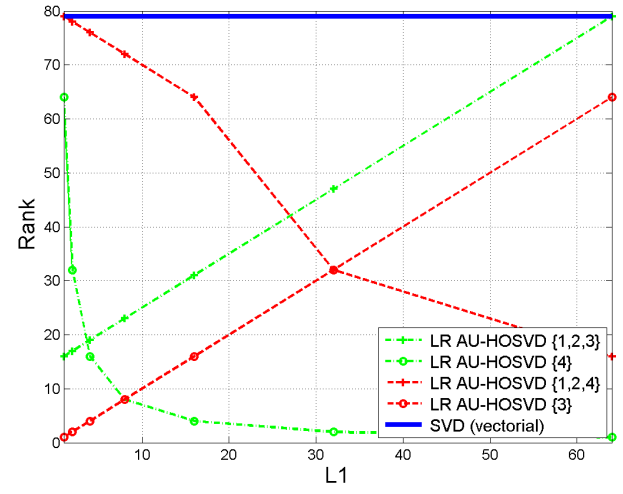

Fig. 3. Vectorial and multimode ranks $\left(r_{1,2,3}, r_{4}, r_{1,2,4}\right.$ and $\left.r_{3}\right)$ of the clutter contribution as a function of $L_{1}$. 


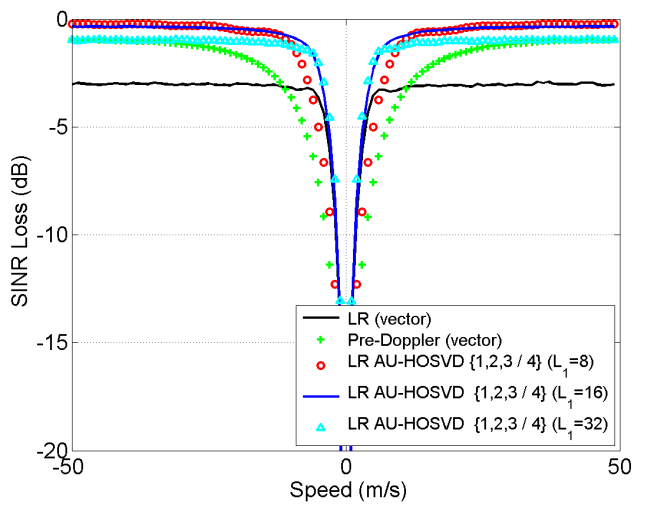

Fig. 4. SINR Losses versus Doppler frequency. Tensorial LR filter built with Partition $\{1,2,3 / 4\} . K=158$. SINR Losses evaluated through 1000 trials.

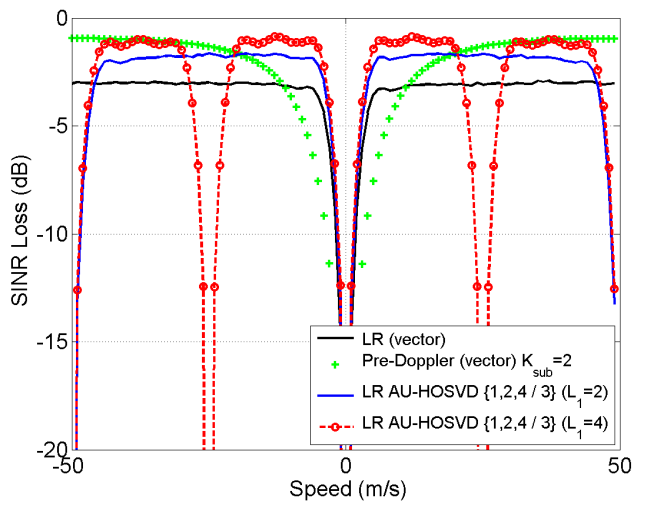

Fig. 5. SINR Losses versus Doppler frequency. Tensorial LR filter built with Partition $\{1,2,4 / 3\}$. $K=158$. SINR Losses evaluated through 1000 trials.

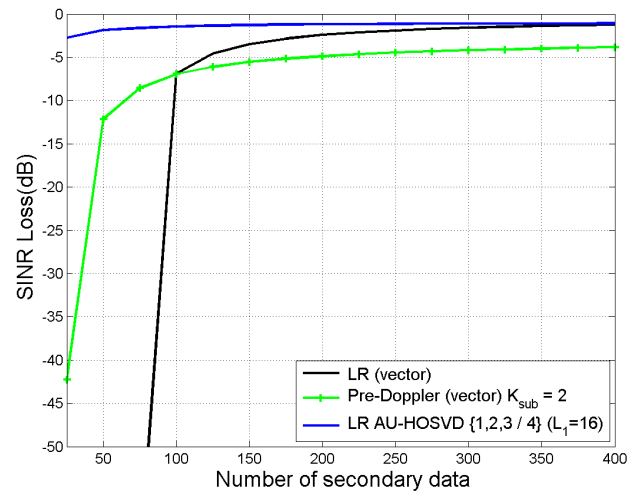

Fig. 6. SINR Losses versus the number of secondary data $(K)$. Tensorial LR filter built with Partition $\{1,2,3 / 4\}$ with $L_{1}=16$. Slow target $\left(f_{s}=0, v=-7 m \cdot s^{-1}\right)$. SINR Losses evaluated through 1000 trials. 


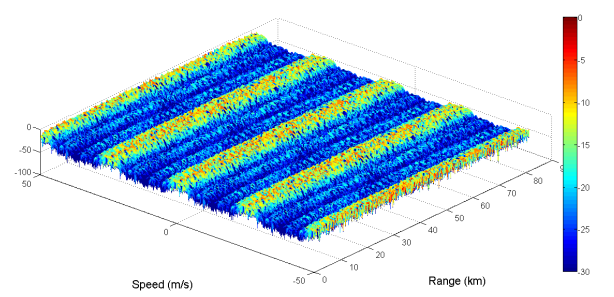

Fig. 7. Range/Doppler map of simulated scene.

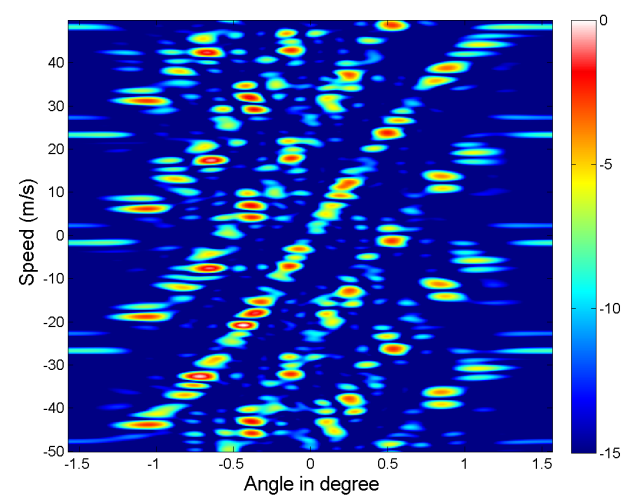

Fig. 8. Angle/Doppler map at the target range of simulated scene.

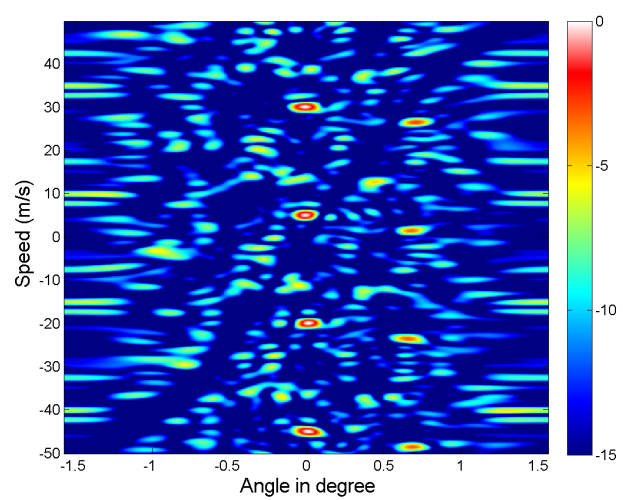

Fig. 9. Angle/Doppler map at the target range after LR vectorial filtering. $K=158$. 


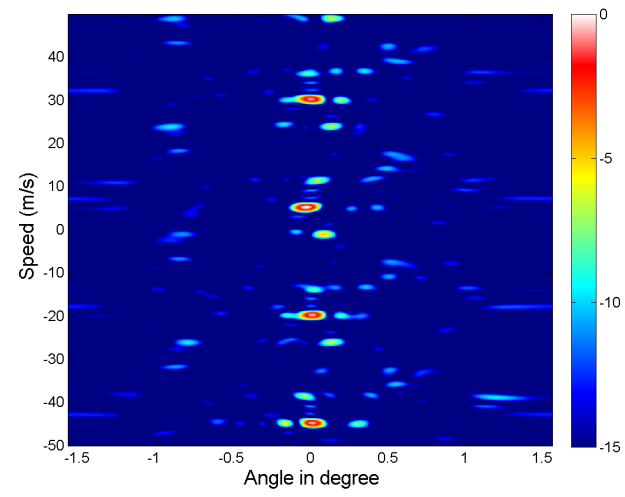

Fig. 10. Angle/Doppler map at the target range after tensorial LR filter built with the partition $\{1,2,3 / 4\}$ with $L_{1}=16$. $K=158$. 